\title{
European Medical Research Group
}

\section{Meeting held 11 July 1994}

The European Medical Research Group met at the Medical Society of London, Lettsom House, London, UK, on 11 July 1994. The guest speaker was Professor TG Dinan of St Bartholomew's Hospital Medical College, London, who gave a lecture on 'Depression and the black humors: new endocrine clues for treatment'.

A poster session was held demonstrating the research in progress of some members of the Group, two of which are published below.

Evaluation of sertraline in overdoses reported in the UK

In the clinical trial development programme of the antidepressant sertraline, a selective serotonin re-uptake inhibitor (SSRI), the drug was shown to have a favourable safety profile. In a database of 1902 patients there were four cases of overdose with sertraline and they were symptomatically managed with no relevant clinical sequelae. ${ }^{1}$

In this paper evaluating the post-marketing experience of sertraline, the overdoses reported to the National Poisons Unit, Guy's Hospital, London from December 1990 to December 1992 are reviewed. The safety of sertraline in overdose was classified according to the fatal toxicity index, psychosis, convulsions, cardiotoxicity, orthostatic hypotension, and sedation, as proposed by de Jonghe and Swinkels. ${ }^{2}$

In this review there were 69 cases where sertraline was taken in combination with at least one other drug or alcohol and 23 reports in which sertraline was taken alone with doses up to $2000 \mathrm{mg}$. In the latter group drowsiness $(\mathrm{n}=5)$ and vomiting $(\mathrm{n}=3)$ occurred most frequently. In the remainder there were no symptoms reported. There were no fatalities in patients taking sertraline alone. In contast to the well known effects of the tricyclic antidepressants, overdose with sertraline did not produce clinically significant reactions such as orthostatic hypotension, cardiotoxicity, psychosis or sedation, or convulsions. This confirms previous experience from the clinical trial development programme, ${ }^{1}$ suggesting a reduced pro-convulsant potential compared to tricyclic antidepressants as presented in a recent review on the clinical use and safety of SSRIs. ${ }^{3}$ These data support the favourable side-effect profile of sertraline, and suggest that it also has a favourable safety margin when taken in overdose.

C BERTI

RA WARD

R BYFIELD

Medical Department, Pfizer Ltd,

Sandwich, Kent, UK

JA HENRY

National Poisons Unit,

Guy's Hospital, London, UK

1 Doogan DP. Toleration and safety of sertraline: experience worldwide. Int Clin Psychophar macol 1991; 6 (suppl 2): 47-56.

2 de Jonghe F, Swinkels JA. The safety of antidepressants. Drugs 1992; 43 (suppl 2): 40-7. antidepressants. Drugs 1902,43 (suppl 2): 40-7. 3 Teicher MH, Glod CA, Cole JO. Antidepressant drugs and the emergence of suicidal

A pilot study of the effect of hormonal pretreatment during human heart transplantation

A study of serial myocardial biopsies from 230 donor hearts during transplantation showed that $39 \%$ of hearts had significant myocardial injury prior to excision. A further $38 \%$ deteriorated significantly during the transplant procedure. Assessment was by quantitative birefringence measurements (QBM). These measure the ability of muscle fibres to respond to adenosine triphosphate (ATP) and calcium and correlate with measurements of cardiac function $(p<0.001)$. Hearts assessed as damaged were worse after transplantation; $51 \%$ vs $4 \%$ needed inotropic agents; of 35 cardiac deaths (donor organ failure, acute rejection, late coronary occlusive disease), 34 occurred in recipients of damaged donor hearts $(p<0.001)$. Hormonal replacement therapy (T3, cortisol and insulin) has been advocated as a method of preventing myocardial injury following brain death. In a prospective randomised study we tested the effect of pretreating 12 donors with $2 \mu \mathrm{g} / \mathrm{h} \mathrm{T} 3,1 \mathrm{~g}$ methylprednisolone and insulin to normalise serum glucose levels. Free T3 levels were abnormal $(2.1 \pm 0.3 \mathrm{pmol})$ and did not normalise $(2.6 \pm 0.6 \mathrm{pmol})$ during pretreatment. No effect was observed on the myocardium QBM $1.35 \pm 0.05$ compared with $1.32 \pm 0.03$ in untreated donors. T3 dosage was increased to $4 \mu \mathrm{g}$ bolus and a continuous infusion $4 \mu \mathrm{g} / \mathrm{h}$. T3 levels normalised after pretreatment ( $\mathrm{n}=11$ donors) and QBM were improved, mean $1.41 \pm 0.06$ as comapred with $1.31 \pm 0.05$ in control group $(p<0.01)$. It is concluded that hormonal treatment of braindead donors appeared to improve myocardial function. Further studies are needed to ascertain whether pretreatment enhances the longterm survival of the recipients.

$M$ CANKOVIK S DARRACOTT-CANKOVIC Department of Clinical Biochemistry, Cambridge University, Cambridge, UK J WALLWORK Transplant Unit, Papworth Hospital, Cambridge CB3 8RE, UK 